\title{
Formulation and Evaluation of Furosemide Solid Self-emulsifying Drug Delivery System
}

\author{
J. Renuka', Y. Ganesh Kumar², D. Saritha ${ }^{3}$, V. Mahesh ${ }^{4}$ \\ 1,4 Department of Pharmaceutics, Vikas College of Pharmaceutical Sciences, Rayanigudem, Nalgonda, \\ Telangana, India, 2,3 Department of Pharmaceutics, Unity College of Pharmacy, Yadadri Bhongir, Telangana, \\ India
}

Abstract

Aim: The objective of the present study was to develop a novel solid self-emulsifying drug delivery system (SEDDS) to enhance the solubility, dissolution rate and ultimately the oral bioavailability of a poorly water-soluble drug, and furosemide. Materials and Methods: Phase solubility studies were conducted using various oils (oleic acid, and soybean oil, sunflower oil, surfactants Tween20, and Cremophor RH 40) and cosolvent (ethanol) for the maximum solubility of furosemide. Ternary phase diagrams were constructed to evaluate the self-emulsification domains and were also for the optimum concentrations of oil and surfactants in the formulation. The globule size analysis, polydispersity and zeta potential of all the developed formulations were studied using Malvern Zetasizer. In vitro release studies and emulsification time were conducted using USP Type II dissolution test apparatus. Statistical Analysis: Fourier transform infrared (FTIR) analysis for investigating the drug excipients interactions and scanning electron microscopy studies for the size, shape, and morphology of the globules after the emulsification process was performed. The formulation of furosemide SEDDS was compared with commercial tablets $\left(\operatorname{Lasix}^{\circledR} 40\right)$. Results: The results of the studies indicated that the rate of dissolution of the developed SEDDS formulations containing furosemide was 2.9-3.6 folds increased compared with that of commercial tablets. The mean globule size $(n=3)$ was observed to be below $100 \mathrm{~nm}$ for the optimized formulations, and the zeta potential was negative which may not interfere in the absorption of the formulation. There were no interactions between the drug, oil, and surfactants, which were confirmed from the results of FTIR studies. Conclusion: The developed SEDDS formulation improved the solubility and in vitro drug release of furosemide when compares with commercial tablet formulation.

Key words: Bioavailability, dissolution, particle size analysis, polydispersity, self-emulsifying, ternary phase, zeta potential

\section{INTRODUCTION}

$\mathrm{F}$ urosemide is a benzoic sulfonamide furan. It is a high ceiling loop diuretic and usually produces an intense diuresis of relatively short duration (4-6 h) with rapid onset $(30 \mathrm{~min})$ that is used for edema and chronic renal insufficiency. Furosemide acts by inhibiting the Na-K-2Cl symporter in the thick ascending limb of the loop of Henle. The bioavailability of furosemide is low due to its low solubility in water $(0.0099 \mathrm{mg} / \mathrm{ml})$, and it is having a bioavailability of $43-69 \%$ after oral administration.

The dissolution rate of poorly water-soluble drugs often becomes a rate-limiting step in their absorption from the gastrointestinal (GI) tract.
Various solubilization methods have been used to increase the drug solubility and dissolution properties including the use of surfactants, water-soluble carriers, polymeric conjugates, and solid dispersions. Among the formulations, self-emulsifying drug delivery system (SEDDS) is a class of emulsion that has received particular attention as a means of enhancing oral bioavailability of the poorly absorbed drug. SEDDS

\section{Address for correspondence: \\ J. Renuka, Department of Pharmaceutics, Vikas College of Pharmaceutical Sciences, Rayanigudem, Nalgonda, \\ Telangana, India. Phone: +91-9741861851. \\ E-mail: chinni2902@gmail.com}

Received: $17-06-2018$

Revised: 25-06-2018

Accepted: 30-06-2018 
is an isotropic mixture of oils and surfactants sometimes including cosurfactants that emulsify under conditions of gentle agitation, similar to those which would be encountered in the GI tract. ${ }^{[1,2]}$

In this study, the potential of lipidic SEDDSs and selfmicroemulsifying drug delivery systems (SMEDDSs) for improving the extent and reproducibility of the oral absorption of furosemide. SEDDSs are defined as isotropic mixtures of natural or synthetic oils, solid or liquid surfactants, or alternatively, one or more hydrophilic solvents, and cosolvents/surfactants. SEDDS typically produce emulsions with a droplet size between 100 and $300 \mathrm{~nm}$ while SMEDDS form transparent microemulsions (ME) with a droplet size of $<50 \mathrm{~nm}$. The small droplet size provides a large interfacial surface area for drug absorption. ${ }^{[3-5]}$

The objective of this study was to develop the optimized formulations of solid SEDDS (S-SEDDS) containing furosemide and evaluate the characteristics of the formulation in vitro. The self-emulsification time in vitro was investigated, and the particle size and zeta potential of the emulsion globules after emulsification were measured, and the bioequivalence study of developed S-SEDDS formulation was investigated compared with the commercial furosemide tablets $\left(\operatorname{Lasix}^{\circledR} 40\right)$.

\section{MATERIALS AND METHODS}

\section{Materials}

Furosemide (drug) was the gift sample from Hetero labs (Hyderabad, India), Tween20 was purchased from Merck Specialities Pvt., Ltd., (Mumbai, India), Cremophor RH 40 was the gift sample from Cognis Gmbh (Monheim, Germany), Oleic acid was purchased from Merck Specialities Pvt., Ltd., (Mumbai, India), Ethyl alcohol purchased from Changshu Yangyuan chemicals (China), and Aerosil purchased from Accord labs (Secunderabad, India). All the chemicals were of analytical grade.

\section{Methods}

\section{Solubility of furosemide in various oils and surfactants}

The solubility study was used to identify the suitable oil and surfactant that possesses good solubilizing capacity for furosemide. $1 \mathrm{ml}$ of each of the selected vehicle was added to each capped vial containing an excess of furosemide. The mixture was heated for $2 \mathrm{~min}$ at $40^{\circ} \mathrm{C}$ in a water bath to facilitate the solubilization using a vortex mixer. Then, the mixtures were shaken with a shaker at $25^{\circ} \mathrm{C}$ for $24 \mathrm{~h}$. After reaching equilibrium, each vial was centrifuged at $4000 \mathrm{rpm}$ for $10 \mathrm{~min}$, and excess insoluble furosemide was discarded by filtration using a membrane filter $(0.45 \mu$,
$13 \mathrm{~mm}$, Whatman, USA). The concentration of furosemide was quantified by ultraviolet (UV) spectrophotometrically at maximum wavelength $274 \mathrm{~nm}$ after relevant dilutions.

\section{Construction of ternary phase diagrams}

The existence of self-emulsifying oil formulation or selfmicroemulsifying oil formulation fields that could selfemulsify or self-microemulsifying under dilution and gentle agitation were identified from ternary phase diagrams of systems containing oil-surfactant cosurfactant. Ternary phase diagrams were constructed using the titration method. The mixture of oil with surfactant or a combination of surfactant and cosurfactant were prepared in different ratios in different conical flasks. A small amount of water at 5\% increment was added into conical flasks. Following each water dilution, the mixtures in conical flasks were vortexed for 2-3 min and were kept aside for $48 \mathrm{~h}$ at room temperature to ensure phase equilibrium. The resulting mixtures were evaluated by visual observation. For the phase diagrams, ME was the region of clear and isotropic solutions that might also contain micelle solutions. Coarse emulsions (CE) were the regions of visibly cloudy dispersions by visual observation. As to the particle size, it is generally held that ME (clear) are $150 \mathrm{~nm}$, while emulsions (visibly cloudy) are in the range of $150-1000 \mathrm{~nm}$. The ternary phase diagrams were constructed using Chemix 3.5 software.

\section{Formulation design of S-SEDDS containing furosemide}

Various formulation designs of SEDDS had been developed using different ratios of various oils, surfactants, and cosurfactant with constant amount of drug. The developed formulations had been shown in Table 1. The formulations were prepared by initially dissolving the formulation amount of furosemide in oil, and then the mixture of surfactant and cosurfactant was added and sonicated for 20 min until a clear concentrate was obtained. The prepared formulations were equilibrated at room temperature for at least $48 \mathrm{~h}$ and observed or examined for signs of turbidity or phase separation before further studies. The prepared formulation was then solidified and filled into the hard gelatin capsules of size " 0. "

\section{Solidification of liquid furosemide SEDDS into S-SEDDS}

Free flowing powders may be obtained from liquid suspoemulsion formulations by adsorption to solid carriers. The adsorption process is simple and just involves the addition of the liquid furosemide formulation on to carrier, i.e., Aerosil (colloidal silicon dioxide) by mixing in a blender. The resulting powder may then be filled directly into capsules. A significant benefit of the adsorption technique is good content uniformity. SEDDS can be adsorbed at high levels (up to $70 \%[\mathrm{w} / \mathrm{w}]$ ) onto suitable carriers. ${ }^{[6,7]}$ 
Table 1: Developed formulations of SEDDS with different ratios of oil, surfactants, and cosurfactants with constant amount of drug

\begin{tabular}{lcccccccc} 
Ingredients & A & B & C & D & E & F & G & H \\
\hline Ethanol and Tween20 (1:1) & $62.50 \%$ & - & - & - & - & - & - & - \\
Ethanol and Tween20 (1:2) & - & $54.16 \%$ & - & - & - & - & - & - \\
Ethanol and Tween20 (1:3) & - & - & $47.05 \%$ & - & - & - & - & - \\
Ethanol and Tween20 (1:4) & - & - & - & $47.05 \%$ & - & - & - & - \\
Ethanol and Cremophor RH 40 (1:1) & - & - & - & - & $66.66 \%$ & - & - & - \\
Ethanol and Cremophor RH 40 (1:2) & - & - & - & - & - & $54.54 \%$ & - & - \\
Ethanol and Cremophor RH 40 (1:3) & - & - & - & - & - & - & $58.33 \%$ & - \\
Ethanol and Cremophor RH 40 (1:4) & - & - & - & - & - & - & - & $57.14 \%$ \\
Oleic acid & $37.50 \%$ & $45.83 \%$ & $52.94 \%$ & $52.94 \%$ & $33.33 \%$ & $45.45 \%$ & $41.66 \%$ & $42.86 \%$ \\
Drug (mg) & 20 & 20 & 20 & 20 & 20 & 20 & 20 & 20 \\
\hline
\end{tabular}

SEDDS: Self-emulsifying drug delivery system

\section{Determination of emulsification time}

The emulsification time, i.e., the time required for the preconcentrate to form a homogeneous mixture on dilution was monitored by visual observation. The emulsification time of SEDDS was determined using USP-dissolution apparatus Type-II (paddle type). Each formulation was added dropwise to $900 \mathrm{ml}$ of purified water at $37^{\circ} \mathrm{C}$ into a stainless steel dissolution paddle rotating at $100 \mathrm{rpm}$ provided gentle agitation. The emulsification time was assessed visually as reported.

\section{Particle size analysis}

The mean particle size and polydispersity index of the size distribution of emulsion globules were determined using photon correlation spectroscopy (which analyses the fluctuations in light scattering due to the Brownian motion of the particles) using Zetasizer 3000 HAS (Malvern Instruments, Malvern, UK) able to measure sizes between 10 and $3000 \mathrm{~nm}$. Light scattering was monitored at $25^{\circ} \mathrm{C}$ at a $90^{\circ}$ angle. The dispersed formulations were measured after dilution (1:100) to produce the required count rate (50-200) to enable the accurate measurement.

\section{Zeta potential measurements}

The zeta potential of SEDDS was determined using Zetasizer 3000 HAS (Malvern Instruments, Malvern, UK). Charge on emulsion droplets, and their mean zeta potential values ( \pm standard deviation) were obtained from the instruments.

\section{In vitro release studies}

The release of furosemide from the S-SEDDS formulation was determined according to USP dissolution apparatus Type-II. To permit the quantitative drug release from S-SEDDS formulation, $900 \mathrm{ml}$ of $0.1 \mathrm{~N} \mathrm{HCl}$ was placed in the dissolution vessel, and then the S-SEDDS formulation filled in hard gelatin capsule was placed in the dissolution medium and was agitated at $100 \mathrm{rpm}$ at $37^{\circ} \mathrm{C}$. At predetermined time intervals of $5,10,15,30,45,60,90$, and 120 min (up to $2 \mathrm{~h}$ ), $5 \mathrm{ml}$ of the samples were withdrawn, and the drug concentration was determined by UV spectrophotometry at $274 \mathrm{~nm}$. The volume removed was replaced each time with fresh dissolution medium. Cumulated released amounts were plotted as a function of time.

\section{Bioequivalence studies}

The bioequivalence studies were performed by comparing the rate and extent of drug release from the developed formulation and the commercial tablet formulation (Lasix ${ }^{\circledR} 40$ ). The release of furosemide from the S-SEDDS formulation and commercial tablet formulation was determined according to USP dissolution apparatus Type-II. The procedure is the same as the in vitro release studies; here, the drug release from the commercial tablet is also determined. ${ }^{[8-13]}$

\section{Fourier transform infrared (FTIR) spectroscopy}

FTIR is the most widely used method in preformulation studies since it can indicate the existence of possible drug excipients interactions in the formulation. In this study, the FTIR instrument was used. FTIR spectra for the drug and the excipients of the optimized formulations were obtained. One part of $\mathrm{KBr}$ was mixed with 100 parts of the optimized formulation and used for the FTIR spectrum. The pure drug was also mixed with $\mathrm{KBr}$ and spectrum was obtained. Both spectra were compared for possible deviations.

\section{Physical stability of preconcentrate}

The optimized formulations $\mathrm{D}$ and $\mathrm{H}$ were stored for 12 weeks and observed for size analysis in the simulated gastric fluid. 


\section{RESULTS AND DISCUSSION}

\section{Solubility of furosemide in various oils and surfactants}

The self-emulsifying formulations consisted of one or more surfactants and drug dissolved in oil. The mixture should be a clear, monophasic liquid at ambient temperature, and should have good solvent properties to allow solubilization of the drug in solution. The solubility's of furosemide in various surfactants and oils are presented in Table 2. From the results of Table 2, among three oils, furosemide appeared to have higher solubility in Oleic acid. Among two surfactants both Tween 20 and Cremophor RH 40 showed better solubility for furosemide. From the above-chosen oils, surfactants and cosurfactants are taken in different ratios for the construction of ternary phase diagrams to know the emulsion and microemulsion domains such that at a particular concentration of oil and surfactant cosurfactant ratios a stable self-emulsifying formulation is formed.

Lipophilic surfactant with Hydrophile-Lipophile Balance (HLB) $<10$ is capable of promoting some emulsification of oil, but the resulting emulsions are normally too crude (in terms of size) to be useful. Hydrophilic surfactants HLB $>10$ are much superior at providing fine, uniform emulsion droplets which are more likely to empty rapidly from the stomach. Furthermore, the large surface area facilitates faster and complete absorption. However, in most cases, it is the right blend of low and high HLB surfactants lead to the formation of stable microemulsion (ME) on exposure to water. Based on these, the high and low HLB surfactants and cosurfactants are chosen for the formulation of furosemide self-emulsifying systems. Oleic acid was chosen as an oil phase for different formulations. The main objective of this experiment is to enhance the solubility of furosemide by formulating SEDDS, so as to decrease the dose and improves the oral bioavailability. $20 \mathrm{mg}$ dose of furosemide was selected to prepare SEDDS.

\section{Construction of ternary phase diagrams}

Figures 1-9 represent the phase diagrams for three different oil-surfactant and cosurfactant water systems: Figures 2-5 (oleic acid, ethanol: Tween 20, and water) and Figures 6-9 (oleic acid, ethanol: CremophoreRH40, and water). These phase diagrams showed the different areas of clear, isotropic $\mathrm{ME}$, and CEs. It can be seen that the largest ME region is seen when the combination of surfactant and cosurfactant is used. When a cosurfactant is added to the system, it further lowers the interfacial tension between the oil and water interface and also influences the interfacial film curvature, which thereby readily deforms around oil droplets. From the phase diagrams, it was observed that as increasing the concentration of surfactant or combination surfactant and cosurfactant increased the self-emulsifying region. Efficiency
Table 2: Solubility of furosemide in various oils, surfactants, and cosurfactants

\section{Surfactants and oils}

Ethanol and tween 20 (1:1)

Ethanol and tween $20(1: 2)$

Ethanol and tween 20 (1:3)

Ethanol and tween 20 (1:4)

Ethanol and Cremophor RH 40 (1:1)

Ethanol and Cremophor RH 40 (1:2)

Ethanol and Cremophor RH 40 (1:3)

Ethanol and Cremophor RH 40 (1:4)

Oleic acid

Soya bean oil

Sunflower oil
Solubility of furosemide in $\mathrm{mg}$

86.6

100.35

120.37

130.2

80.8

113.62

136.78

149.68

4.01

1.98

0.884

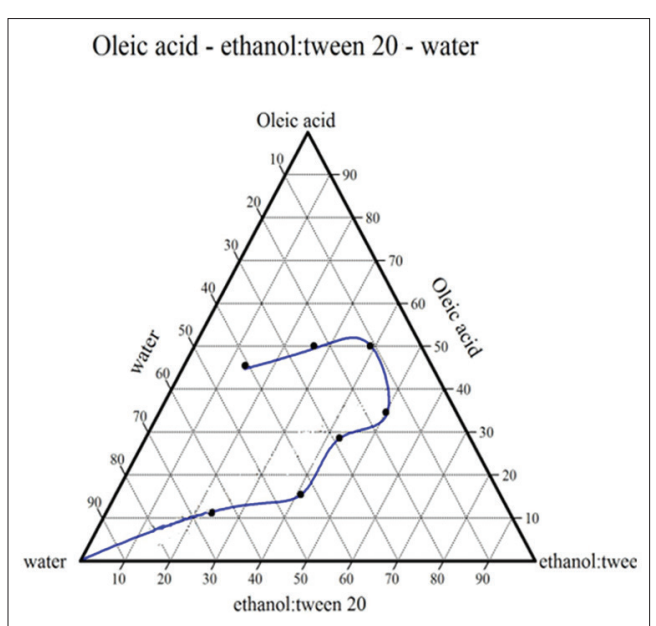

Figure 1: Ternary phase diagram for oleic acid, ethanol: Tween20 (1:1), and water

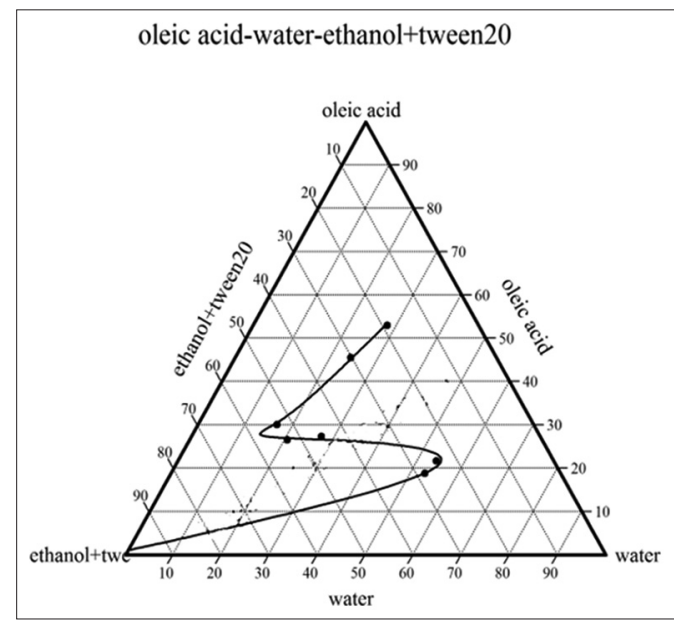

Figure 2: Ternary phase diagram for ethanol: Tween20 (1:2), water

of self-emulsification was good when the surfactant concentration was more than $50 \%$. 


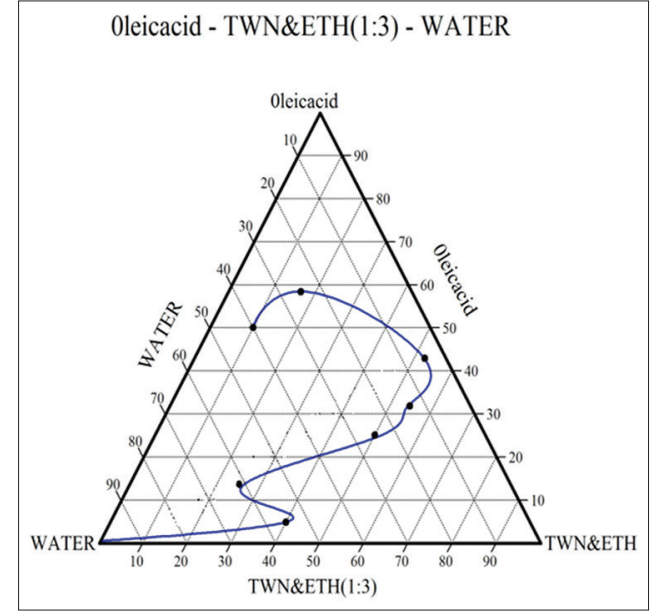

Figure 3: Ternary phase diagram for oleic acid, ethanol: Tween20 (1:3), and water

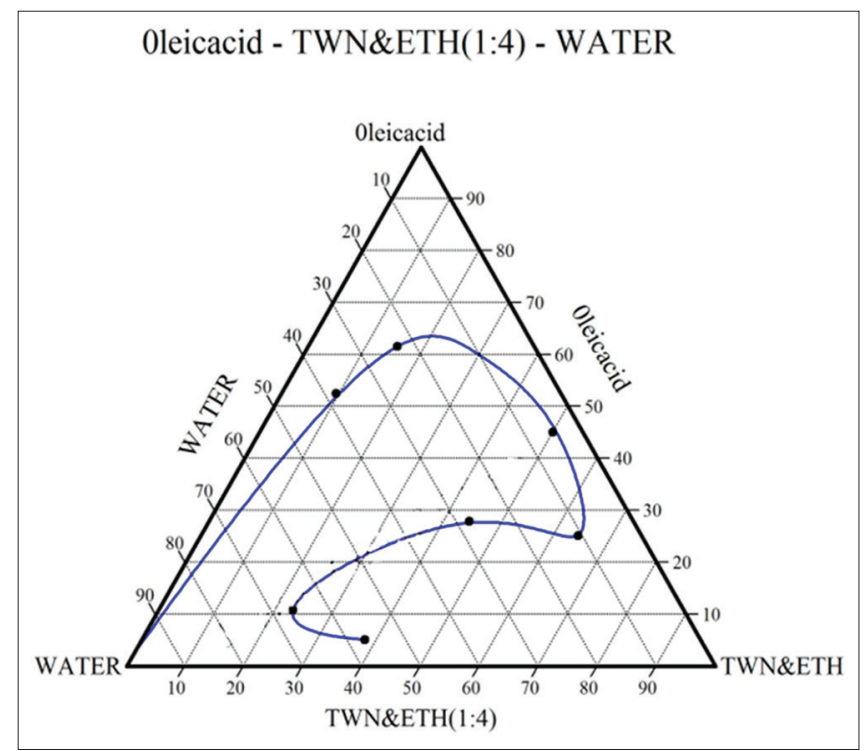

Figure 4: Ternary phase diagram for oleic acid, ethanol: Tween20 (1:4), and water

\section{Determination of emulsification time}

SEDDS form fine oil in water emulsions with gentle agitation on their introduction into aqueous media. Since the free energy required to form an emulsion is very low, the formation is thermodynamically spontaneous. The rate of emulsification is an important index for the assessment of the efficiency of emulsification that is SEDDS should disperse completely and quickly when subjected to aqueous media under mild agitation. A time of 2 min was used as an evaluation index in the emulsification process. To evaluate the emulsification time for the formulations prepared were conducted both in acidic medium $(0.1 \mathrm{~N} \mathrm{HCl})$ and water to simulate the range of conditions encountered in GI tract.

From the results in Table 3, the minimal emulsification time was found with the formulations F (49 s), G (57 s), H (53 s), and $\mathrm{D}(58 \mathrm{~s})$. The highest emulsification time was found to

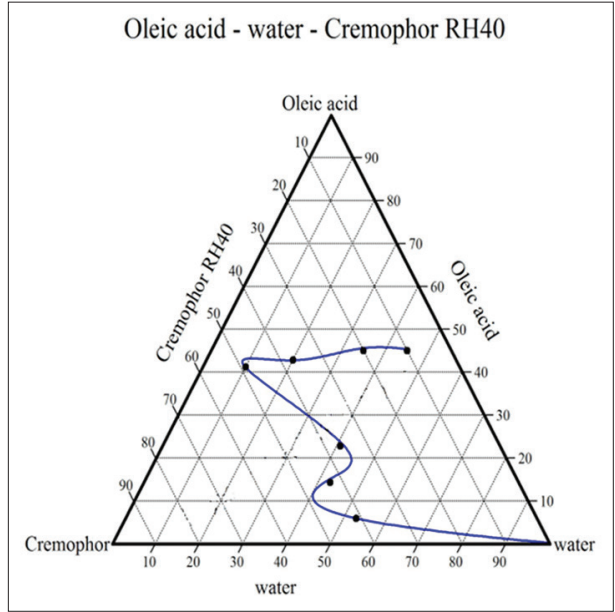

Figure 5: Ternary phase diagram for oleic acid, ethanol:Cremophor RH40 (1:1), and water

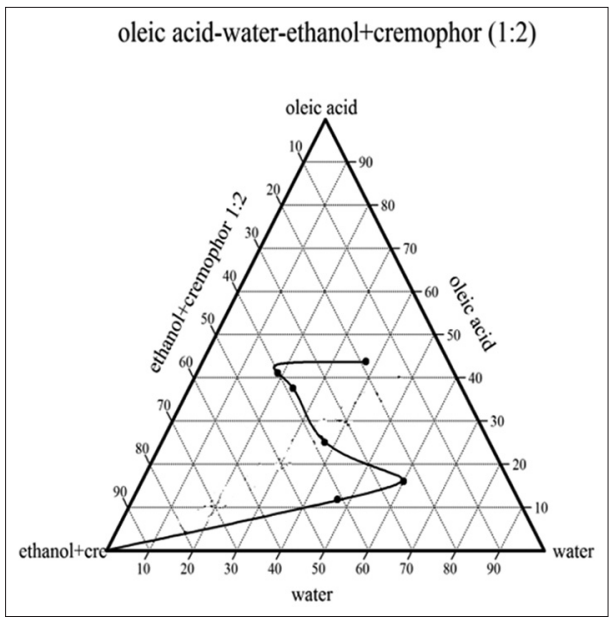

Figure 6: Ternary phase diagram for oleic acid, ethanol:Cremophor RH40 (1:2), and water

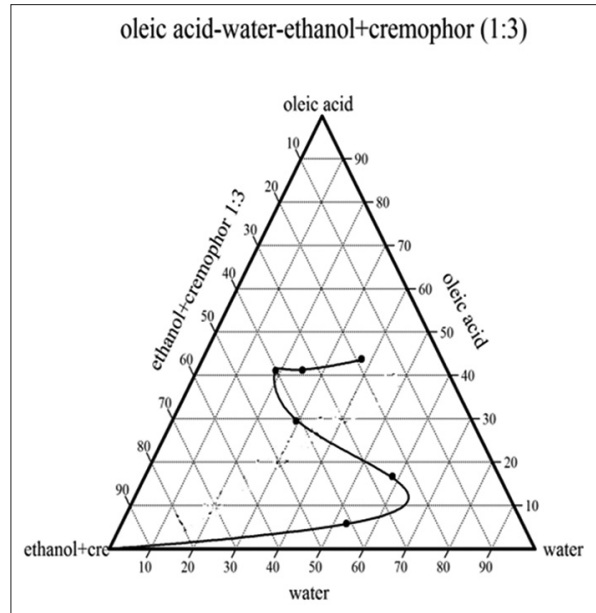

Figure 7: Ternary phase diagram for oleic acid, ethanol:Cremophor RH40 (1:4), and water

be in the formulations A ( $83 \mathrm{~s})$ this may be due to the high viscosity of Tween 20 and if the viscosity of the oil and cosurfactant used is also high, or the content of Tween 20 


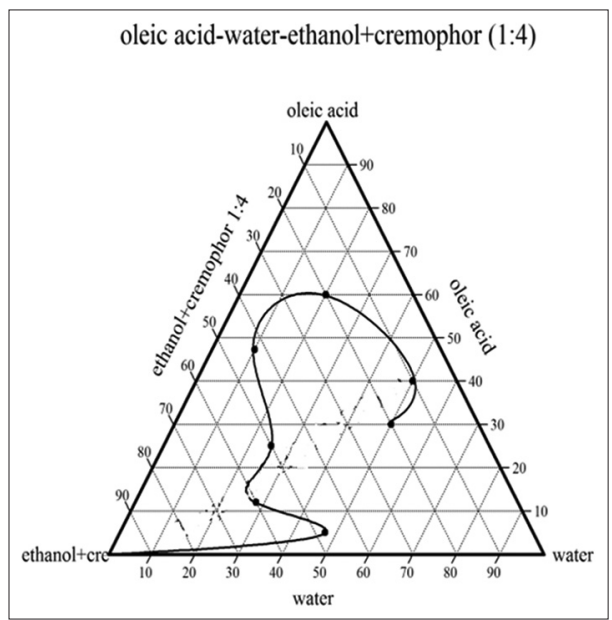

Figure 8: Ternary phase diagram for oleic acid, ethanol:Cremophor RH40 (1:3), and water

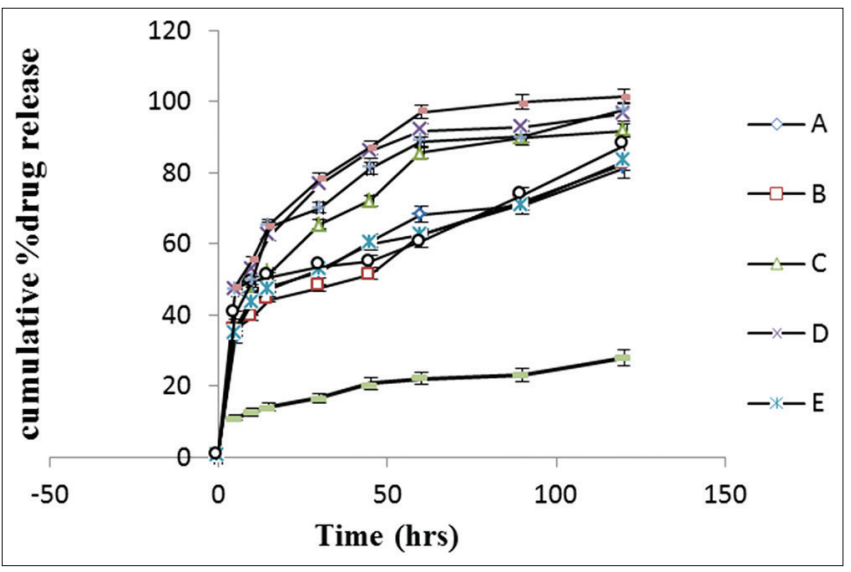

Figure 9: Graph showing cumulative percent of drug release from various formulations

Table 3: Emulsification times of various SEDDS formulations in $0.1 \mathrm{~N} \mathrm{HCl}$ and Distilled water (D.W)

\begin{tabular}{lcc} 
Formulation & $\begin{array}{c}\text { Emulsification } \\
\text { time (s) 0.1 N HCl }\end{array}$ & $\begin{array}{c}\text { Emulsification } \\
\text { time (s) D.W }\end{array}$ \\
\hline A & 83 & 92 \\
B & 71 & 84 \\
C & 62 & 73 \\
D & 58 & 59 \\
E & 66 & 70 \\
F & 49 & 51 \\
G & 57 & 60 \\
H & 53 & 59 \\
\hline
\end{tabular}

SEDDS: Self-emulsifying drug delivery system

is very high will lead to a low emulsification rate and the emulsification time will be greater. Tween 20 had the highest viscosity of all the excipients used in the preparation of the formulation. The viscosity of the formulations increased with the increase in Tween 20 that can be seen visually. When the content of Tween 20 increased from $30 \%$ to $60 \%$, the emulsification time tended to decrease first and then increase. The emulsification time is low for the formulation with Tween 20 in low concentration and increased as the content of Tween 20 is increased. In the formulations having maximum emulsification time resulted in the formation of gel-like structures on dilution with aqueous media and required at least one more minute to fully disperse the system. In the formulas H (49 s), G (57 s), and H (53 s), the emulsification time is less compared with the other formulas this may be due to the low viscosity of the surfactant CremophoreRH40.

There are two factors that affect the emulsification time of SEDDS: One is the free energy of the system and other is the viscosity of the system. The free energy may have been more important than viscosity in the emulsification process. Although the formulations have low viscosity, the spontaneous formation of the interface between the oil and water phases was energetically unfavorable resulting in the low rate of self-emulsification. The tendency to emulsify spontaneously and also the progress of emulsion droplets were observed. The tendency to form an emulsion was judged as "good" when droplets spread easily in water and formed a fine milky solution emulsion, and it was judged "bad" when there was poor or no emulsion formation with immediate coalescence of oil droplets, especially when stirring was stopped.

\section{Particle size analysis}

Droplet size of the emulsion is a crucial factor in selfemulsification/dispersion performance because it determines the rate and extent of drug release as well as absorption. Smaller droplets formed give an increased surface area to release form. The reduction of droplet size to values below $100 \mathrm{~nm}$ leads to the formation of SMEDDS, which is stable, isotropic and clear o/w dispersions.

Self-emulsifying systems were properly diluted and are used for droplet size analysis using Zetasizer 3000 HAS (Malvern Instruments, UK). Average droplet size and polydispersity index were determined.

The least particle size was observed in the formulations $\mathrm{D}$ and $\mathrm{H}$, in which the cosurfactant and surfactant concentrations are taken in the ratio of 1:4 (i.e. Ethanol:Tween20 (1:4) in formulation D and ethanol:CremophoreRH40 (1:4) in formulation $\mathrm{H}$ ) and the remaining formulations the particle size were observed to be large it is because of low surfactant concentration compared to formulas $\mathrm{D}$ and $\mathrm{H}$. The particle size of the formulations A-D and E-H decreased gradually with the increase of surfactant concentration. The particle size of droplets is low with the formulations prepared with CremophoreRH40 compared with formulations prepared with Tween20; this is because of low viscosity of CremophoreRH40.

The mean particle size of the formulation $\mathrm{D}$, containing oleic acid as oil, ethanol as co-surfactant, and Tween 20 as 
surfactant (i.e., in 1:4 ratio) found to be $85.50 \mathrm{~nm}$ and in the formulation $\mathrm{H}$, containing oleic acid as oil, ethanol as cosurfactant, and CremophoreRH40 as surfactant (i.e., in 1:4 ratio) found to be $67.49 \mathrm{~nm}$, such as decrease in the droplet size might be the result of more surfactant being available to stabilize the oil-water interface. Furthermore, the decrease in the droplet size reflects the formation of a better closepacked film of surfactant at the oil-water interface, thereby stabilizing the oil droplets.

Zeta potential of all the SEDDS formulation is found to be a negative charge. The mean particle size and Zeta potential are given in Table 4.

\section{In vitro release studies}

In vitro dissolution studies were performed for all the formulations developed; using USP-dissolution apparatus Type II paddle method. The SEDDS containing $20 \mathrm{mg}$ of furosemide was filled into hard gelatin capsules and introduced into the dissolution medium $(0.1 \mathrm{~N} \mathrm{HCl})$ and maintained the temperature at $3^{\circ} \mathrm{C}$. The revolution speed of the paddle was kept constant at $100 \mathrm{rpm}$ throughout the dissolution studies. The samples of $5 \mathrm{ml}$ were withdrawn at time intervals of 5 , $10,15,30,45,60,90$, and $120 \mathrm{~min}$, filtered the samples using Whatman filter paper. The concentration of furosemide was determined spectrophotometrically at $274 \mathrm{~nm}$.

From all the developed formulations, the percent release of furosemide from the formulations $\mathrm{D}, \mathrm{G}$, and $\mathrm{H}$ was found to be higher than the other formulations developed and the commercial formulation (Lasix ${ }^{\circledR} 40 \mathrm{mg}$ ). The percentage released after $2 \mathrm{~h}$ was $96.47 \%, 97.89 \%$, and 101.44 for $\mathrm{D}$, $\mathrm{G}$, and $\mathrm{H}$, respectively. $27.86 \%$ release was observed with the commercial formulations after $2 \mathrm{~h}$. It could suggest that furosemide dissolved perfectly in SEDDS formulation and could be released due to its small droplet size which permits a faster rate of drug release into the aqueous phase. The release of SEDDS from formulation $\mathrm{H}$ (mean droplet size $67.49 \mathrm{~nm}$ ) was found to be more than formulation D (mean droplet size $85.50 \mathrm{~nm}$ ). Cumulative percent drug release is high in formulation $\mathrm{H}$ because of low viscosity and high concentration of surfactant, i.e., Cremophor RH 40.

From the results, it is considered that the performance of SEDDS depends on two factors: One is the ability of the self-emulsifying mixture to form an emulsion with uniform

\begin{tabular}{ccc}
\multicolumn{2}{c}{$\begin{array}{c}\text { Table 4: Mean particle size and Zeta potential of } \\
\text { developed formulations ( } \mathrm{D} \text { and H) }\end{array}$} \\
Formulation & $\begin{array}{c}\text { Mean droplet } \\
\text { size (nm) }\end{array}$ & $\begin{array}{c}\text { Zeta } \\
\text { potential (MV) }\end{array}$ \\
\hline D & 85.50 & -0.979 \\
H & 67.49 & -4.36 \\
\hline
\end{tabular}

SEDDS: Self-emulsifying drug delivery system fine particle size droplets and the second is the ability of SEDDS to present the furosemide in a solubilized and highly dispersed form, thereby overcoming dissolution step.

Based on the self-emulsification process, particle size data and stability of emulsions and in vitro dissolution profiles the formulations $\mathrm{D}, \mathrm{G}$, and $\mathrm{H}$ are selected for the further studies.

\section{FTIR spectroscopy}

FTIR analysis of all the excipients used in the formulation and the drug was studied for the interaction of the excipients and the drug in the formulation. Here, in this study, it was observed that there is no significant interaction which was observed according to the graphs produced.

\section{CONCLUSION}

SEDDSs containing furosemide had been developed for enhancing the oral bioavailability, which was confirmed, by the in vitro drug release of developed formulation and the commercial tablet (Lasix ${ }^{\circledR} 40$ ). The solubility of furosemide was examined in various oils, surfactants, and cosurfactants from the solubility results, Tween 20, Cremophor RH 40, oleic acid, and ethanol were selected as surfactants, oil, and cosurfactant or cosolvent. Individual phase diagrams of oilsurfactant: Cosurfactant water were constructed and observed that the ME region was high when the cosurfactant is added, and the optimum concentrations of three components from the phase diagrams were taken for formulating SEDDS. Emulsification time was accessed for all the formulations and was found to be less for the formulations $\mathrm{D}, \mathrm{G}$, and $\mathrm{H}$. As the concentration of surfactant was increased there was an increase in the emulsification time this may be due to the increase in the viscosity of the surfactant. The globule sizes of all the formulations were evaluated. The mean particle size of the formulation D, containing oleic acid as oil, ethanol as cosurfactant, and Tween 20 as surfactant (i.e., in 1:4 ratio) found to be $85.50 \mathrm{~nm}$ and in the formulation $\mathrm{H}$, containing oleic acid as oil, ethanol as cosurfactant, and Cremophor RH 40 as surfactant (i.e., in 1:4 ratio) found to be $67.49 \mathrm{~nm}$. FTIR analysis of the excipients, drug, and formulation shows no drug excipients interactions. There was no effect on droplet size in the formulations $\mathrm{D}$ and $\mathrm{H}$ as observed for 12 weeks of stability studies. The in vitro dissolution rate of furosemide from SEDDS was significantly increased from 2.9 to 3.6 folds compared with that from the commercial tablet (Lasix $\left.{ }^{\circledR} 40\right)$.

\section{REFERENCES}

1. Maeda T, Takenaka H, Yamahira Y, Noguchi T. Use of rabbits for GI drug absorption studies: Relationship between dissolution rate and bioavailability of griseofulvin tablets. J Pharm Sci 1979;68:1286-9. 
2. Chiba Y, Kohri N, Iseki K, Miyazaki K. Improvement of dissolution and bioavailability for mebendazole, an agent for human echinococcosis, by preparing solid dispersion with polyethylene glycol. Chem Pharm Bull 1991;39:2158-60.

3. Kislalioglu MS, Khan MA, Blount C, Goettsch RW, Bolton S. Physical characterization and dissolution properties of ibuprofen: Eudragit coprecipitates. J Pharm Sci 1991;80:799-804.

4. Moyano JR, Arias MJ, Gines JM, Rabasco AM. Dissolution behavior of oxazepam in presence of cyclodextrins-evaluation of oxazepam-dimeb binary system. Drug Dev Ind Pharm 1997;23:379-85.

5. OkonogiS, OguchiT, YonemochiE, Puttipipatkhachorn S, Yamamoto K. Improved dissolution of ofloxacin via solid dispersion. Int J Pharm 1997;156:175-80.

6. Pouton CW. Formulation of self-emulsifying drug delivery systems. Adv Drug Deliv Rev 1997;25:47-58.

7. Gursoy RN, Benita S. Self-emulsifying drug delivery systems (SEDDS) for improved oral delivery of lipophilic drugs. Biomed Pharmacother 2004;58:173-82.

8. Wakerly MG, Pouton CW, Meakin BJ, Morton FS. Selfemulsification of vegetable oil-non-ionic surfactant mixtures. ACS Symp Series 1986;311:242-55.

9. Charman SA, Charman WN, Rogge MC, Wilson TD, Dutko FJ, Pouton CW. Self-emulsifying drug delivery systems: Formulation and biopharmaceutic evaluation of an investigational lipophilic compound. Pharm Res 1992;9:87-93.

10. Shah NH, Carvajal MT, Patel CI, Infeld MH, Malick AW. Self-emulsifying drug delivery systems (SEDDS) with polyglycolized glycerides for improving in-vitro dissolution and oral absorption of lipophilic drugs. Int $\mathrm{J}$ Pharm 1994;106:15-23.

11. Pouton CW. SEDDS: Assessment of the efficiency of emulsification. Int J Pharm 1985;27:335-48.

12. Tang B, Cheng G, Gu JC, Xu CH. Development of solid self-emulsifying drug delivery systems: Preparation techniques and dosage forms. Drug Discov Today 2008;13:606-12.

13. Pouton CW. Formulation of poorly water-soluble drugs for oral administration: Physicochemical and physiological issues and the lipid formulation classification system. Eur J Pharm Sci 2006;29:278-87.

Source of Support: Nil. Conflict of Interest: None declared. 